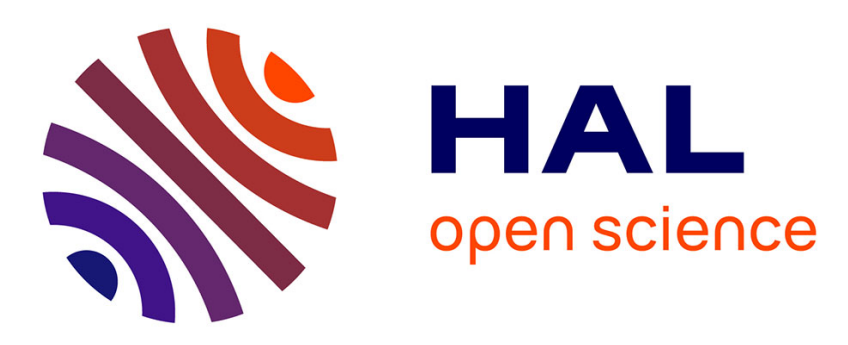

\title{
Efficiency of Sequential Spatial Adaptive Sampling Algorithm to Accelerate Multi-Frequency Near-Field Scanning Measurement
}

Sébastien Serpaud, Alexandre Boyer, Sonia Ben Dhia, Fabio Coccetti

\section{To cite this version:}

Sébastien Serpaud, Alexandre Boyer, Sonia Ben Dhia, Fabio Coccetti. Efficiency of Sequential Spatial Adaptive Sampling Algorithm to Accelerate Multi-Frequency Near-Field Scanning Measurement. IEEE Transactions on Electromagnetic Compatibility, 2022, 64 (3), pp.816 - 826. 10.1109/TEMC.2021.3136096 . hal-03543405

\author{
HAL Id: hal-03543405 \\ https://hal.laas.fr/hal-03543405
}

Submitted on 26 Jan 2022

HAL is a multi-disciplinary open access archive for the deposit and dissemination of scientific research documents, whether they are published or not. The documents may come from teaching and research institutions in France or abroad, or from public or private research centers.
L'archive ouverte pluridisciplinaire HAL, est destinée au dépôt et à la diffusion de documents scientifiques de niveau recherche, publiés ou non, émanant des établissements d'enseignement et de recherche français ou étrangers, des laboratoires publics ou privés. 


\title{
Efficiency of Sequential Spatial Adaptive Sampling Algorithm to Accelerate Multi- Frequency Near-Field Scanning Measurement
}

\author{
Sébastien Serpaud, Student Member, IEEE, Alexandre Boyer, Sonia Ben Dhia, and Fabio Coccetti
}

\begin{abstract}
Near-field scanning is an attractive method to diagnose conducted and radiated emission issues at board or circuit level. However, the long measurement time to scan a dense electronic board at numerous frequencies is a major drawback that limits its growth in industry. To overcome this problem, methods to accelerate the measurement, such as the sequential spatial adaptive sampling algorithm, must be found. This method was developed to speed up a near-field scans measured in frequency domain at only one frequency. The proposed paper capitalizes on this method to extend its principle and demonstrate its effectiveness for near-field scans measured at multiple frequencies. Several measurement strategies and frequency segmentation approaches are compared. A definition of the best strategy and approach to allow a time reduction factor of almost one order magnitude compared to the full sampling measurement has been proposed.
\end{abstract}

Keywords - Electromagnetic compatibility (EMC), near-field scanning (NFS), adaptive sampling, time optimization.

\begin{tabular}{|c|c|c|}
\hline Parameter & Definition & Unit \\
\hline Ax, Ay & $\begin{array}{l}\text { Size in 2D plane along } \mathrm{x} \text { axis and } \mathrm{y} \text { axis of space } \\
\text { domain of measurement. }\end{array}$ & {$[\mathrm{mm}, \mathrm{ml}$} \\
\hline$d$ & Final resolution of the full sampling map & [mm] \\
\hline$D$ & Total number of points of the full sampling map & \\
\hline$F$ & $\begin{array}{l}\text { Represents a regionalized variable representing the } \\
\text { near-field radiated by the device under test. }\end{array}$ & {$[\mathrm{dBA} / \mathrm{m}]$} \\
\hline$F\left(p_{i}\right)$ & Represents an achievement of $F$ at the position $p_{i}$. & {$[\mathrm{dBA} / \mathrm{m}]$} \\
\hline$\hat{F}\left(p_{i}\right)$ & Estimation of $F$ value at the position $p_{i}$. & {$[\mathrm{dBA} / \mathrm{m}]$} \\
\hline$F_{M}$ & Final measurement dataset of $F$. & {$[\mathrm{dBA} / \mathrm{m}]$} \\
\hline$F_{N}$ & Initial dataset of $F$ collected during the first step. & {$[\mathrm{dBA} / \mathrm{m}]$} \\
\hline$M$ & $\begin{array}{l}\text { Total number of points where } F \text { is measured after } \\
\text { SSAS process. }\end{array}$ & \\
\hline$N$ & Number of points captured during the first step. & \\
\hline$N_{B W}$ & $\begin{array}{l}\text { Number of frequencies band for the selected } \\
\text { approach. }\end{array}$ & \\
\hline NFreq $q_{\text {Captured }}$ & Total number of frequencies captured on $P_{M}$. & \\
\hline$N_{\text {Freq }}$ & Number of frequencies captured of $F\left(N_{\text {Freq }}=1601\right)$. & \\
\hline$N q$ & Number of points captured during the step $q=\{2 . . \mathrm{Q}\}$ & \\
\hline$\Omega$ & Space domain of measurement. & \\
\hline$p_{q i}$ & $\mathrm{i}^{\text {nd }}$ position evaluated during the step $\mathrm{q}$. & $\{x, y\}$ \\
\hline$P_{c}\left(p_{q i}\right)$ & Local dataset of the $N c$ points neighboring $p_{q i}$. & $\{x, y\}^{N c}$ \\
\hline
\end{tabular}

These results have been obtained in the framework of the IRT Saint Exupery's research projects: ROBUSTESSE and FELINE. We acknowledge the financial and in-kind support (background knowledge and services) from the IRT Saint Exupery's industrial and academic members and the financial support of the French National Research Agency.

\begin{tabular}{clc}
$P_{M}$ & Final dataset of the $M$ points where $F$ is measured. & $\{x, y\}^{M}$ \\
$P_{N}$ & Initial dataset of the $N$ points where $F$ is measured & $\{x, y\}^{N}$ \\
$Q$ & during the first step. & \\
Speed & Total number of steps. & Speed motion of the probe (fixed at $0.02 \mathrm{~m} . \mathrm{s})$ \\
$T_{\text {algo }}$ & Total time to compute the SSAS algorithm. & {$[\mathrm{s}]$} \\
$T_{c}$ & Unit time to configure receiver (fixed at $0.2 \mathrm{~s})$ & {$[\mathrm{s}]$} \\
$T_{\text {conf }}$ & Total time to configure receiver during measurement & {$[\mathrm{s}]$} \\
$T_{\text {meas }}$ & Total time to capture $F_{M}$. & {$[\mathrm{s}]$} \\
$T_{\text {move }}$ & Total time to move probe on $P_{M}$. & {$[\mathrm{s}]$} \\
$T_{M}$ & Total time to capture $F$ on $P_{M}$ position. & {$[\mathrm{s}]$} \\
$W$ & Initial sampling step. & {$[\mathrm{mm}]$} \\
\hline \hline
\end{tabular}

\section{INTRODUCTION}

WITH the current industrial economic constraints, it is necessary to evaluate electromagnetic behavior of an electronic device as early as possible in its development process. If simulation tools fail to predict accurately the electromagnetic compatibility (EMC) of a complex equipment, the EMC engineer must have efficient measurement-based investigation tools. The near-field scanning (NFS) method applied on electronic devices has been developed for several years to meet this need. This contactless measurement is carried out above an electronic Device Under Test (DUT) without the need to implement specific design constraints. Moreover, measurement results with high reproducibility $(<1 \mathrm{~dB}$ for the stationary process in frequency domain) is ensured by the intrinsically weak sensitivity of the experimental read-out of the equipment set-up configuration.

Despite the aforementioned key features offered by the NFS method, a limiting factor, hindering a wider industrial deployment of this approach, is the effective scanning time duration. In the context of investigation activities, measuring only one field component $\left(\mathrm{H}_{\mathrm{z}}\right)$ can be sufficient to identify the root cause of an EMC related issue. A complete characterization of the near-field emission above a complex printed circuit board (PCB) in a large frequency range may last several tens of hours [1]. Moreover, in the context of modeling activities, the minimization of the number of points required to

S. Serpaud, F Coccetti are with the IRT Saint-Exupéry institute, Toulouse, France (e-mail: sebastien.serpaud@irt-saintexupery.com, Fabio.coccetti@irtsaintexupery.com)

S. Serpaud, S. Ben-Dhia and A. Boyer are with LAAS, Univ. de Toulouse, INSA, Toulouse, France (e-mail: sonia.bendhia@insa-toulouse.fr, alexandre.boyer@laas.fr) 
create a model from NFS measurement could be interesting as presented by [2]. The probe displacement duration and the processing time associated to the control of the near-field scanner affects the measurement time. However, the main contribution is the time required by the receiver to capture the voltage across the near-field probe (which may reach up to 75 $\%$ of the total measurement time as show in Fig. 9). It is directly linked to the acquisition speed and the number of captured points. The most straightforward strategy to reduce the measurement time consists in decreasing the number of points.

An effective method to reach this goal is to use a spatial adaptive sampling, which selects spatial sampling position only where a sufficient amount of information can be collected. Several approaches have been proposed for electromagnetic applications [3] [4] [5]. For a near-field scanning above a PCB, the approach described in [6] [7] [8], dedicated to adaptive sampling of planar surface, ensures a significant reduction of the number of samples. However, the massive use of Kriging model and the random sampling lead to a significant increase of the time due to the algorithm computation and the probe movements. The Sequential Spatial Adaptive Sampling (SSAS), presented in [1] and [9], solves these limitations, which reduces by a factor 5 to 13 the total measurement time compared to the full sampling approach. Compared to other method, the SSAS algorithm is very low time consuming and is initialized from two set-up parameters (probe dimension and scans height). It does not require a specific adjustment of the algorithm parameters.

Nevertheless, all these approaches [6] [7] [3] [4] [5] [8] [1] [9] were applied to reduce the acquisition time of monofrequency near-field maps. In a practical EMC investigation campaign, where electronic devices produce wide band electromagnetic emission, a mono-frequency scanning can be insufficient since one measurement frequency has to be selected carefully. EMC problems have often to be analyzed at a wide frequency range to be efficient. The purpose of this paper is to extend the SSAS algorithm presented in [1] to the multifrequency near-field scanning and determine the configuration that optimizes the measurement time reduction. Two strategies for applying the SSAS algorithm are discussed and completed by an evaluation of four frequency-segmentation approaches.

The paper is organized as follows: Section II summarizes the principle of the SSAS algorithm developed for mono-frequency measurements. Section III describes how the SSAS algorithm is extended to the multi-frequency domain. The method is validated on a case study described in Section IV. Section V presents the results, where the influence of algorithm parameters on measurement time reduction are discussed. All the symbols used to describe the algorithm are summarized in the list of symbols.

\section{Principles of the SeQuential Spatial Adaptive SAMPLING ALGORITHM}

This section gives the main principles of the SSAS algorithm used in this paper. A detailed description is provided in [1]. The method was developed and validated for NFS measurements done at only one frequency. As proposed by the IEC standard [10], the magnitude of near-field emission $F$ is captured by a near-field probe at a constant height $h_{\text {meas }}$ above a device under test. $F$ is defined on a rectangular planar surface $\Omega$ that is sampling on a regular grid at a predefined resolution $d$.

In order to limit the total measurement time, the purpose of this algorithm is to capture a limited number of positions in $\Omega$ allowing a complete reconstruction of the magnitude distribution of $F$ in $\Omega$. The selection criterion of SSAS algorithm selects these positions to ensure a reconstruction of $F$ without any significant loss of information [1].

The method relies on a Multi-Level Adaptive approach [11] which starts with an initial collection: $F_{N}$, offering a sparse meshing of the scanning surface $\Omega$. The $F_{N}$ is made only from two measurement parameters known by user: the probe dimension: $r_{\text {loop }}$ and the height of measurement: $h_{\text {meas }}$. Then, the SSAS algorithm increases the meshing locally according to a selection criterion. A new sampling point is added when the neighbor measurement points are above the noise floor and if a sufficient field gradient is observed (5). Depending on the selection criterion, either the measurement time reduction or the accuracy of the field reconstruction is favored. Finally, the full grid near-field map with the final resolution $d$ is reconstructed by spatial interpolation. The different steps of the proposed method are resumed in the following parts.

\section{A. The final resolution of the reconstructed near-field map}

The final near-field emission map is the sampling of the field $F$ on a rectangular space domain $\Omega$, meshed with according to a regular sampling approach. The sampling step $d$ defines the final resolution of the map. For a given scanning surface $\Omega$, a fine resolution leads to an increase in the number of points. Although the choice of the map resolution is user-dependent, it cannot be better than the near-field probe resolution. A commonly accepted rule-of-thumb is that a magnetic probe resolution is equal to the radius $r_{\text {Loop }}$ of the near-field probe. The lower value of the final resolution $d$ will be set to the probe radius value, as given by (1).

$$
d=r_{\text {loop }}
$$

\section{B. The sequential spatial adaptive sampling}

The SSAS algorithm is based on the Multi-Level Adaptive approach [11] initially introduced in [9] and [1]. The first step consists in collecting an initial dataset: $F_{N}$ on the Sukharev regular sampling grid of points over $\Omega$ separated by $W$. The objective is to map $\Omega$ with a limited number of points by an optimal spatial cover without any a priori knowledge about $F$. This initial dataset forms also a regular sampling grid, but with a sampling step $W$ larger than $d$. The choice of $W$ has a serious impact on the optimality of the final solution. If it is too large, relevant information on $F$ may be lost. In contrast, a too small value of $W$ will lead to a large number of initial samples and therefore a weak reduction of the measurement time. Once the initial dataset of measurement points has been collected, the scanning surface $\Omega$ is progressively meshed after $(Q-1)$ measurement steps, as illustrated in Fig. 1. 


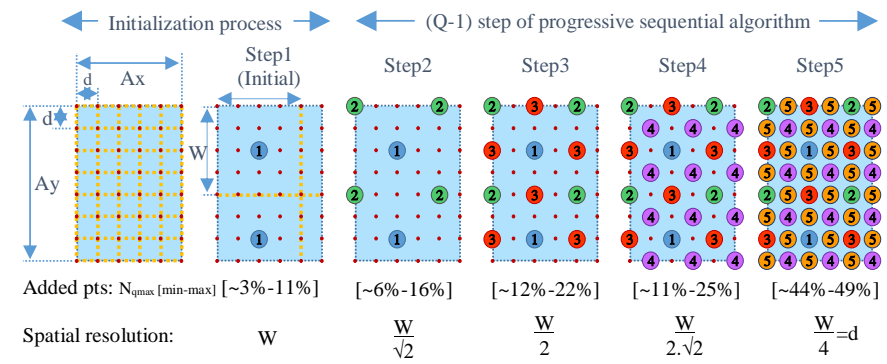

Fig. 1. Spatial adaptive sampling where $\Omega=\{\mathrm{Ax}, \mathrm{Ay}\}$ is progressively covered in 5 steps according to the two input parameters : $\mathrm{d}$ and $\mathrm{W}$

The sampling resolution starts step by step with a value of $W$ an then decreases linearly up to the $d$ value. The number of steps $Q$ and the initial sampling resolution $W$ are defined from the setup parameters according to (2) and (3) respectively. (4) gives the number of points $N$ collected during the initial step.

$$
\begin{array}{r}
Q \leq 2 \cdot\left\lfloor\log _{2}\left(h_{\text {meas }} / r_{\text {loop }}\right)\right\rfloor+3 \\
W=\mathrm{d} \cdot 2^{(Q-1) / 2} \\
\left.N=\left[\frac{A_{x}}{W / d}-0.5\right\rceil \cdot \mid \frac{A_{y}}{W / d}-0.5\right\rceil
\end{array}
$$

\section{Selection criterion}

After gathering of the initial dataset $F_{N}$, the adaptive part of the SSAS algorithm refines the sampling step by step $(q=2 . . \mathrm{Q})$, according to the definition of a selection criterion. The objective is to minimize the total number of measured points $M$, by ensuring that the final set of $M$ measurements of $F$ allows building a good estimator that minimizes the prediction error of $F$ at any position on $\Omega$. During the $\mathrm{q}^{\text {th }}$ measurement step, a maximum number of points $N q_{\max }$ can be measured. The selection criterion validates whether the field value on the point $p_{q i} F\left(p_{q i}\right)$ must be measured in order to minimize $N q$, the actual number of points captured during the $\mathrm{q}^{\text {th }}$ measurement iteration.

This selection criterion, given by (5), is based on the standard deviation approach calculated from a local estimator $\hat{F}\left(p_{q i}\right)$. $\hat{F}\left(p_{q i}\right)$ estimates $F$ at the position $p_{q i}$ from known values $P_{C}\left(p_{q i}\right)$ of $F$. The point $p_{q i}$ is captured only if the field deviation (in $\mathrm{dB}$ ) between $\hat{F}\left(p_{q i}\right)$ and $F\left(p_{c}\right)$ is larger than $k$ [in $\left.\mathrm{dB}\right] . k$ denotes the maximum tolerated deviation of the field, between a point and its neighbors in $M$, to ensure an exact reconstruction of $F$. With a relevant definition of $k$, the acquisition of noisy points can be eliminated. For more information about the definition of the selection criterion and its ability to capture only the most relevant information, the reader is invited to read the previous paper [1].

$$
\exists p_{c} \in P_{M}^{\prime}|| d B\left(F\left(p_{c}\right)\right)-d B\left(\hat{F}\left(p_{q i}, f_{v}\right)\right) \mid>k
$$

The selection criterion requires a local interpolator of the field $\hat{F}$. Let $P_{M}^{\prime}=\left\{p_{m}\right\}_{m=1}^{\dot{M}} \subset P_{M}$ the temporary dataset resuming all the measurement positions of $F$ already captured. $P_{C}\left(p_{q i}\right)=\left\{p_{c}\right\}_{c=1}^{N_{c}} \subset P_{M}^{\prime}$ denotes a local dataset including all the positions neighboring $p_{q i}$ as shown in Fig. 2. The Inverse Distance Weighting (IDW) interpolator is a widely used method for spatial estimation (6) (7). Its very short computing time makes it a good choice to compute $\widehat{F}\left(p_{q i}\right)$. In [1], the weighting coefficient value $p$ was fixed at 3 to increase the effect of the closest points of $p_{q i}$.

$$
\widehat{F}\left(p_{q i}\right)=\sum_{j=1}^{N_{c}} \lambda_{j}\left(p_{q i}\right) \cdot F_{c}\left(p_{j}\right)
$$

Where:

$$
\lambda_{j}\left(p_{q i}\right)=\frac{w_{j}\left(p_{q i}\right)}{\sum_{l=1}^{N_{C}} w_{l}\left(p_{q i}\right)} \text { and } w_{u}\left(p_{q i}\right)=\left(\frac{1}{d\left(p_{u}, p_{q i}\right)}\right)^{p} .
$$

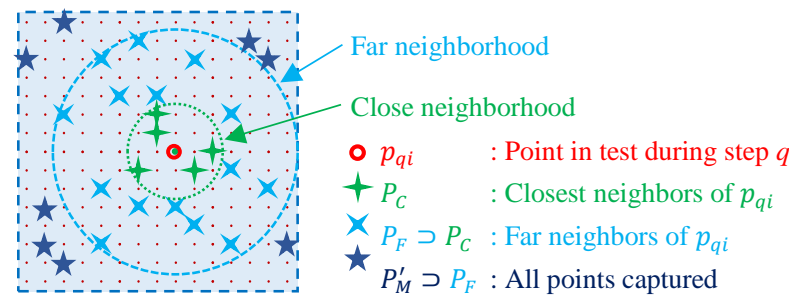

Fig. 2. Definition of the datasets of points in the neighbourhood of $p_{q i}$

In (5), if $k$ value is low, the probability to capture $p_{q i}$ is high. Otherwise, if $k$ value increases, the probability to capture $p_{q i}$ decreases. Three definitions of $k$ parameter have been proposed and justified in [1]. They offer different levels of compromise between measurement time reduction and accuracy of the field reconstruction:

- The first limit definition, $k_{l}$, is a simple constant (8). It provides the largest reduction of the number of measurement points but with a price of inaccurate field reconstruction around the emission map maxima.

- $k_{2}$ is an improvement of $k_{1}$ definition to adjust the limit according to the field value on neighbor points (9). This limit definition increases the precision around the position of the maximum emission level of the map. However, it may lead to inaccurate field reconstruction around the local secondary maxima of the near-field emission map.

- $\quad k_{3}$ overcomes the inaccuracies introduced by $k_{2}$ and refines the spatial meshing around the primary and all secondary maxima of the near-field emission map (10). However, among the three criteria, it leads to the smallest reduction of the number of measurement points.

$k_{1}=10[d B]$

$k_{2}=1+9 \cdot \sqrt{\frac{\max \left(F_{M}^{\prime}\right)-\hat{F}\left(p_{q i}\right)}{\max \left(F_{M}^{\prime}\right)-\min \left(F_{M}^{\prime}\right)}}[d B]$

$k_{3}=1+9$.

$$
\sqrt{\frac{\max \left(F_{M}^{\prime}\right)-\hat{F}\left(p_{q i}\right)}{\max \left(F_{M}^{\prime}\right)-\min \left(F_{M}^{\prime}\right)} \cdot \min \left(1, \sqrt{\frac{\max \left(F_{F}\left(p_{q i}\right)\right)-\hat{F}\left(p_{q i}\right)}{10}}\right)}[d B]
$$

Where: $\hat{F}\left(p_{q i}\right) \leq \max \left(F_{F}\left(p_{q i}\right)\right) \leq \max \left(F_{M}^{\prime}\right)$.

\section{The interpolator for the final emission map reconstruction}

After the SSAS algorithm process, $M$ points irregularly distributed on $\Omega$ have been captured. To reconstruct the nearfield map with the final resolution $d$, it is necessary to interpolate the field from the $F_{M}$ dataset captured in $\Omega$. The 
evaluation of the spatial sampling is linked to the performance of the interpolator. The better is the interpolation, the more the spatial sampling constraints can be relaxed. Thus, the number of samples will be reduced. The Kriging interpolation method was retained as an efficient spatial unbiased estimator with minimum variance. The Scikit library on Python [15] has been used to process the adaptive meshing interpolation. Exponential kernel is chosen to initialize the interpolator.

\section{EXTENSION OF THE SSAS ALGORITHM TO MULTI- FREQUENCY DOMAIN}

In a multi-frequency measurement context, $F$ is a vector of $N_{\text {Freq }}$ value of the near-field at each frequency: $f_{v=1 . . N_{F r e q}}$ where $N_{\text {Freq }}$ is the number of frequencies. The objective, of the SSAS algorithm, is to collect $F$ at all $N_{\text {Freq }}$ frequencies for all $M$ points. Two strategies may be applied to collect the $F_{N}$ dataset.

In the follow up of this work, the word 'strategy' defines how the algorithm covers all position points and all frequency values. The first strategy is to process all frequency sub-ranges for each $P_{N}$ positions while for the second strategy all $P_{N}$ positions are processed for each frequency sub-range. The main difference between both approaches is the order to process the $P_{N}$ positions and the frequency vector: $f_{v=1 . . N_{F r e q}}$. The selected strategy could have a significant impact on the measurement time. In the first strategy, called « Point-by-Point », the number of receiver reconfigurations is increased since it is done $N$ times for each point. In contrast, the number of probe displacements is increased in the second strategy, called « Range-by-Range », because all points are processed $N_{\text {Freq }}$ times for each frequency range. One strategy will be more efficient than the other depending on the receiver configuration time and the probe displacement time. Section V.B discusses about this point. The first strategy is assumed in the next paragraphs of this chapter.

The next paragraphs discuss about the way to find the best segmentation of the frequency range. In the context of harmonic emission, two samples, very close each other in the frequency domain, are statistically linked to the same source. Consequently, two maps at two close frequencies are statistically equal spatially speaking. In EMC field, the frequency range of measurement could be spread over several decades. In order to improve the frequency resolution, the frequency range is often split into several sub-ranges. An advantage to split the frequency range is to adapt the measurement parameters (e.g. the frequency resolution and the resolution bandwidth filter RBW) according to the frequency in order to limit the measurement time. Following the sub-ranges parameters, it is possible to define the total time to capture a frequency range according to (11). The $a$ parameter is relative to the choice of some parameters as the type of filter (Peak, QPeak, average...), the average post-processing factor...

$$
\text { SwpTime }_{B W}=a \cdot \frac{S P A N}{R B W^{2}}
$$

In the follow up of this work, the word 'approach' defines how the frequency range is split. The next chapters present four different approaches that could be used to optimize the measurement time. Section $\mathrm{V}$ gives a performance analysis of each approach.

\section{A. A-Approach-Approach based on the full frequency range measurement}

The SSAS algorithm presented in [1] is developed only for mono-frequency measurements. A basic approach to extend it to multi-frequency measurements is to update the selection criterion to take into account the frequency vector of $F$. The selection criterion resumed in (5) can be renamed by $S C\left(p_{q i}, f_{v}\right)$ for the specific frequency $f_{v}$ of $F$ measurement. As a reminder, if $\operatorname{SC}\left(p_{q i}, f_{v}\right)$ is true, the $F$ value at the frequency $f_{v}$ must be captured at the position $p_{q i}$. The new selection criterion $S C\left(p_{q i}\right)$ defines the condition to capture a frequency range ( $N_{\text {Freq }}$ points of frequency) of $F$ at the position $p_{q i}$. It can be resumed by the below equation (12), where $V$ is the "OR" Boolean operator. With this selection criterion, a frequency range of $F$ is captured at the $p_{q i}$ position when the frequency selection criterion is true at least at one frequency.

$$
\operatorname{SC}\left(p_{q i}\right)=\mathrm{V}_{v=1}^{N_{F r e q}} \operatorname{SC}\left(p_{q i}, f_{v}\right)
$$

In (12) the selection criterion for a frequency range is the result of several computations ( $N_{\text {Freq }}$ times) of the selection criterion at each frequency value of this range. Consequently, the selection criterion in a large frequency range is not impacted by the noise level variation over the same frequency band.

The $\hat{F}\left(p_{q i}\right)$ computation is involved to execute two simple algorithms. The first is the neighbor search algorithm used to collect all values of $F$ in $P_{M}^{\prime}$ dataset already captured around $p_{q i}$. It can be executed one time for the full frequency range. The second is the IDW algorithm resumed in (6). This algorithm is very low time consuming even if it is computed for $N_{\text {Freq }}$ frequencies. Therefore, even with this new selection criterion, the SSAS algorithm process time is low.

The A-Approach is not optimal. A measurement of the full frequency range can be required, according to the selection criterion, just because significant emission levels could be present at only one frequency. Consequently, a lot of time is wasted in capturing a low noise emission level for the remaining frequencies.

\section{B. B-Approach-Approach based on the spectrum of emission of DUT}

Another approach can be proposed to solve the aforementioned shortcoming. In order to limit Tmeas, it could be relevant to split the analysis over a large frequency range. The probability that each source radiates at a specific frequency range is high, especially on a complex PCB that includes numerous components (e.g. power converter, low frequency microcontroller, high frequency FPGA). The frequency range segmentation following the different activities running on the DUT, as proposed by [12], could be an interesting approach.

The objective is to focus the measurement on the specific activity location thus to reduce the width of the frequency range captured for all points. The gain of the B-Approach is based on 
two hypotheses. First, the DUT presents several sources with different harmonic frequency profile activities. The frequency spectrum must be wide or narrow band but it must be limited in a specific frequency range. Second, each source is scattered over limited areas above the DUT.

The difficulty of the B-Approach is to define the good number and the boundary of all frequency sub-ranges. An analysis of the initial $F_{N}$ dataset (e.g. from [12]) should define the optimal number of the frequency sub-ranges according to the frequency distribution of the sources on the DUT. This one would require a complex algorithm that hamper the deployment of this approach.

\section{C-Approach-Approach based on standard test frequency segmentation}

Some equipment, generally the oldest, have a limited number of points (e.g. 201/401/801/1601 points). Hence, the wider the frequency band of interest, the coarser will be the segmentation. Moreover, the finer the resolution demanded at both, high and low frequencies, the finer the segmentation necessary of the entire frequency band must be. Consequently, EMC emission standards tests (e.g. [10] [18]) provide guidelines about on how to carry out this frequency segmentation. The C-Approach proposes to reuse the frequency segmentation proposed by the EMC standards tests. This approach has the same advantage than the previous one without the need of a complex algorithm.

\section{D-Approach-Approach based on frequency-by-} frequency segmentation

In the two previous approaches, the objective is to focus the measurement on a limited number of sub-ranges in order to reduce the time spent to capture $F$ at the frequencies where no emission is observed. The last evaluated approach is to process the SSAS algorithm frequency by frequency as initially proposed in [1]. For each $N_{\text {Freq }}$ frequency, the SSAS algorithm optimizes the number of points that must be captured according to the frequency selection criterion in frequency: $S C\left(p_{q i}, f_{v}\right)$.

According to [1], for the steps $(q=2 . . Q)$, the SSAS algorithm measures only the field at the specific frequencies and positions that carry the most important information. Therefore, the DApproach proposes the optimal measurement time: $T_{\text {meas }}$. Except during the initial step, no time is wasted to measure the noise level or insignificant value of $F$.

However, the limitation of this approach is related to $T_{\text {move }}$. The length of path used to cover all points at each frequency run and at each step $q=2 . . Q$ is significantly increased. Another limitation is related to the SSAS algorithm computation time: $T_{\text {algo }}$. In this approach, the selection criterion is computed $N_{\text {Freq }}$ times for each point, at each frequency run and at each step.

\section{PResentation of The VAlidation CASe StUdy: "FPGA"}

Fig. 3 presents the XILINX Spartan 6 FPGA device test board. Two types of magnetic field probe are used: one for the $H x$ and $H y$ components and another for the $H z$ component. The spatial resolution of the probes is $0.5 \mathrm{~mm}$. The probes are moved at $1.1 \mathrm{~mm}$ above the FPGA package. The height of measurement is fixed at $h_{\text {meas }}=1.85 \mathrm{~mm}$ above the top of the IC substrate. More information is presented in [13].

The frequency range of the magnetic near-field measurements starts at $5 \mathrm{MHz}$ and increases up to $1.8 \mathrm{GHz}$. Three frequency sub-ranges are defined to cover the whole frequency band as resumed in Table I. The total time to collect all $N_{\text {Freq }}$ frequency values of $F$ is equal to 2.25 seconds per point.

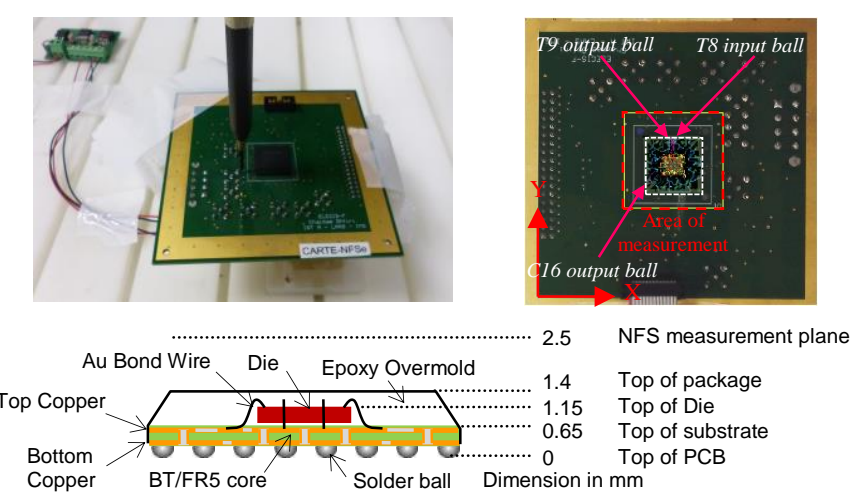

Fig. 3. Description of case study : "FPGA"

Table I : Frequency Sub-range Parameters

\begin{tabular}{|c|c|c|c|c|c|c|c|}
\hline $\begin{array}{l}\text { Sub- } \\
\text { range }\end{array}$ & $\begin{array}{c}\text { Fmin } \\
{[\mathrm{MHz}]}\end{array}$ & $\begin{array}{c}\text { Fmax } \\
{[\mathrm{MHz}]}\end{array}$ & $\begin{array}{l}\text { SPAN } \\
{[\mathrm{MHz}]}\end{array}$ & $\mathrm{a}$ & $\begin{array}{l}\mathrm{RBW} \\
{[\mathrm{KHz}]}\end{array}$ & $N_{F R E Q}$ & $\begin{array}{c}\text { SwpTime } \\
{[\mathrm{s}]}\end{array}$ \\
\hline BW1 & 5 & 50 & 45 & 1 & 10 & 400 & 0.45 \\
\hline BW2 & 50 & 500 & 450 & 1 & 30 & 400 & 0.5 \\
\hline \multirow[t]{2}{*}{ BW3 } & 500 & 1800 & 1300 & 10 & 100 & 801 & 1.3 \\
\hline & & & & & Total: & 1601 & 2.25 \\
\hline
\end{tabular}

It is possible to define, for this case study, the theoretical optimal parameters of $Q$ and $W$ parameters according to (2), (3) and the input parameters, as summarized in Table II.

Table II : SSAS Algorithm Parameters and Time Definition

\begin{tabular}{llccc}
\hline \multicolumn{1}{c}{ Parameters } & \multicolumn{3}{c}{ Values } & Unit \\
\hline Space domain of measurement $\Omega$ & $\{A x, A y\}$ & $\{29,28\}$ & {$[\mathrm{mm}, \mathrm{mm}]$} \\
Final resolution & $d=r_{\text {Loop }}$ & 0.5 & {$[\mathrm{~mm}]$} \\
G. & $h_{\text {meas }}$ & 1.85 & {$[\mathrm{~mm}]$} \\
Height measurement & $D$ & 3363 & {$[\mathrm{pts}]$} \\
Number of points of full sampling grid & $Q$ & 5 & {$[\mathrm{step}]$} \\
Number of steps & $W$ & 2 & {$[\mathrm{~mm}]$} \\
\& & $W$ Initial resolution & $N$ & 210 & {$[\mathrm{pts}]$} \\
\hline Number of points collected at first step &
\end{tabular}

\section{A. Analysis of the case study activity}

In a previous paper [13], an analysis of this case study activity in frequency domain was carried out and it is resumed in Fig. 4. The analysis identified three typical frequency profiles activities that could be localized in specific areas above the FPGA as shown in Fig. 4. Both input and output switching activities are much closer (in both spatial and frequency domains) and could be resumed in one activity. For more details, all data measurements are available [17] for noncommercial and academic use only.

\section{B. The approaches definition according to the case study characteristics}

Based on the demonstrator activity, the frequency range can be divided into 2 sub-ranges for the B-Approach. The near-field scans, on the first sub-range (below $100 \mathrm{MHz}$ ), displays all FPGA activities. In this case, the gain is limited. However, the near-field scans, on the second sub-range (upper than $100 \mathrm{MHz}$ ) 


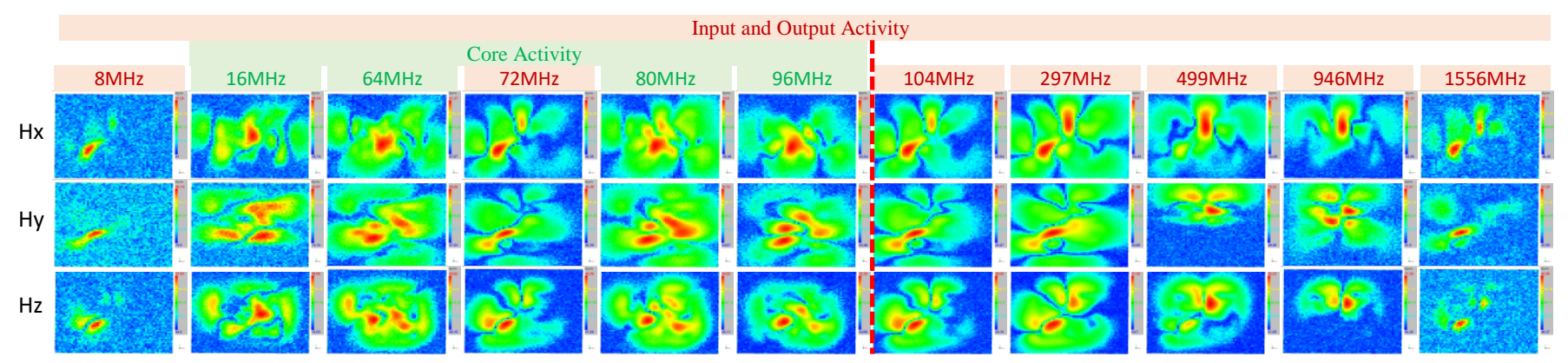

Fig. 4. Example of near-field scans in frequency domain for each FPGA activities

display mainly the IO activity in specific areas. This is the largest frequency range and the source locations are limited to the IO positions.
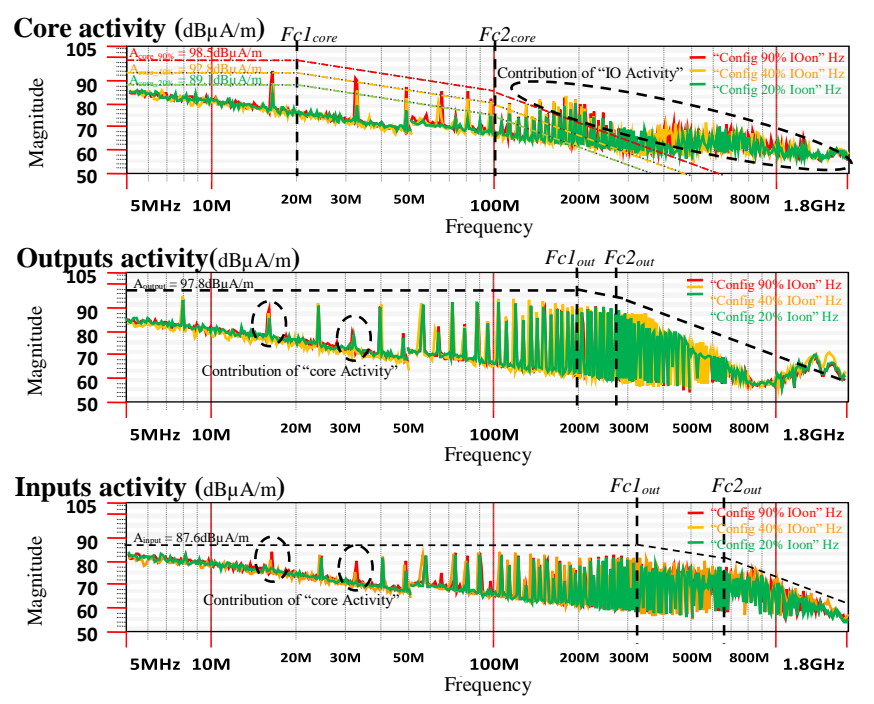

Fig. 5. Spectrum of internal FPGA activities (Hz measurement)

According to the Table I, the frequency range can be divided into 3 sub-ranges for the C-Approach and 1601 sub-ranges for the D-Approach. Table III resumes the frequency range segmentation for the four proposed approaches.

Table III : Frequency Range Segmentation by Approach

\begin{tabular}{|c|c|c|c|c|c|c|}
\hline \multirow[b]{2}{*}{ Approach } & \multirow[b]{2}{*}{$N_{B W}$} & \multirow[b]{2}{*}{ Sub-range } & \multicolumn{4}{|c|}{ Receiver parameters } \\
\hline & & & \multicolumn{2}{|c|}{$[\mathrm{MHz}]$} & $\begin{array}{l}N_{F r e q} \\
\text { [Nb Freq] }\end{array}$ & $\begin{array}{c}\text { SwpTime }_{B W} \\
{[\mathrm{~s}]}\end{array}$ \\
\hline A & 1 & BW1 & 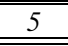 & 1800 & $N_{\text {Freq }}=1601$ & 2.25 \\
\hline \multirow{3}{*}{ B } & \multirow{3}{*}{2} & BW2.1 & 5 & 100 & 450 & 0.50 \\
\hline & & BW2.2 & 100 & 1800 & 1051 & 1.75 \\
\hline & & & & Total: & $N_{\text {Freq }}=1601$ & 2.25 \\
\hline \multirow{4}{*}{$\mathrm{C}$} & \multirow{4}{*}{3} & BW3.1 & 5 & 50 & 400 & 0.45 \\
\hline & & BW3.2 & 50 & 500 & 400 & 0.50 \\
\hline & & BW3.3 & 500 & 1800 & 801 & 1.3 \\
\hline & & & & Total: & $N_{\text {Freq }}=1601$ & 2.25 \\
\hline $\mathrm{D}$ & 1601 & BW4.1.1601 & 5 & 1800 & $N_{\text {Free }}=1601$ & 2.25 \\
\hline
\end{tabular}

\section{VALIDATION OF THE PROPOSED SSAS ALGORITHM}

The next chapter presents the time reduction of each approach for both proposed strategies. The first part of this section considers that the measurement is done without accounting for $T_{\text {algo }}$ and the equipment configuration time: $T_{\text {conf }}$. In the second part, the equipment configuration time is introduced and the full time of the measurement is evaluated.
The final chapter discuss about the measurement error added by the SSAS algorithm according to the aforementioned approaches and strategies.

\section{A. Measurement time evaluation}

Let $T_{\text {move }}$ be the time to move the probe between each position and $T_{\text {meas }}$ be the time to capture $F$ value at each position for all the $N_{\text {Freq }}$ frequency values. Let $T_{N}$ be the time to collect the initial dataset (according to the first strategy) and $T_{M}$ be the total time to process the SSAS algorithm on $\Omega$ according to the equations (13)-(23). In these equations, the ' $N$ ' subscript index denotes the time during the first step, the ' $N Q$ ' subscript index denotes the time during the SSAS steps $(q=2 . . Q)$ and the ' $M$ ' subscript index denotes the total time for all steps. $N_{\text {Freq }}$ Captured denotes the sum of frequencies captured of $F$ at each position (16) (17). $N_{\text {Freq }}$ Captured is directly linked to $T_{\text {meas }_{M}}$ value (19). Table IV resumes the values relating to the selected case study.

$$
\begin{aligned}
& T_{\text {move }_{N}}=\frac{(N-1) \cdot W}{\text { Speed }^{*}} \\
& T_{\text {move }_{M}}=T_{\text {move }_{N}}+T_{\text {move }_{N Q}} \\
& N_{\text {Freq }} \text { Captured }_{N}=N . N_{\text {Freq }} \\
& N_{\text {Freq }_{\text {Captured }} Q}=\sum_{b w=1}^{N_{B W}}\left[N_{\text {Freq }_{B W}} \cdot \sum_{q=2}^{Q} N q_{B W}\right] \\
& N_{\text {Freq }_{\text {Captured }_{M}}}=N_{\text {Freq }_{\text {Captured }_{N}}}+N_{\text {Freq }_{\text {Captured }_{N Q}}} \\
& T_{\text {meas }_{N}}=N \cdot \text { SwpTime } \\
& T_{\text {meas }_{M}}=N_{\text {Freq }_{\text {Captured }_{M}}} \cdot\left(\frac{\text { SwpTime }}{N_{\text {Freq }}}\right) \\
& T_{N}{ }^{\prime}=T_{\text {move }_{N}}+T_{\text {meas }_{N}} \\
& T_{\text {conf }_{N}}=N \cdot N_{B W} \cdot T_{C} \\
& T_{\text {conf }_{M}}=T_{\text {conf }_{N}}+T_{\text {conf }_{N Q}} \\
& T_{M}=T_{\text {algo }_{M}}+T_{\text {move }_{M}}+T_{\text {conf }_{M}}+T_{\text {meas }_{M}}
\end{aligned}
$$

Where $T_{c}$ denotes the time to configure the receiver for a selected bandwidth ( $T_{C}$ is fixed at $0.2 \mathrm{~s}$ for this study). $N q_{B W}$ denotes the number of points actually captured during each $N_{B W}$ frequency run (with $N q=\sum_{b w=1}^{N_{B W}} N q_{B W}$ ). It is worth noting that $T_{\text {move }_{N}}$ and $T_{\text {conf }_{N}}$ depend on the used strategy. (13) - (23) are defined for the "Point-By-Point" strategy. For the "Rangeby-Range" strategy, $N_{B W}$ multiply (13) and $N$ divide (21).

Fig. 6 shows the number of points collected for each 
Table IV : SSAS Performance Indicators for the Selected Case Study

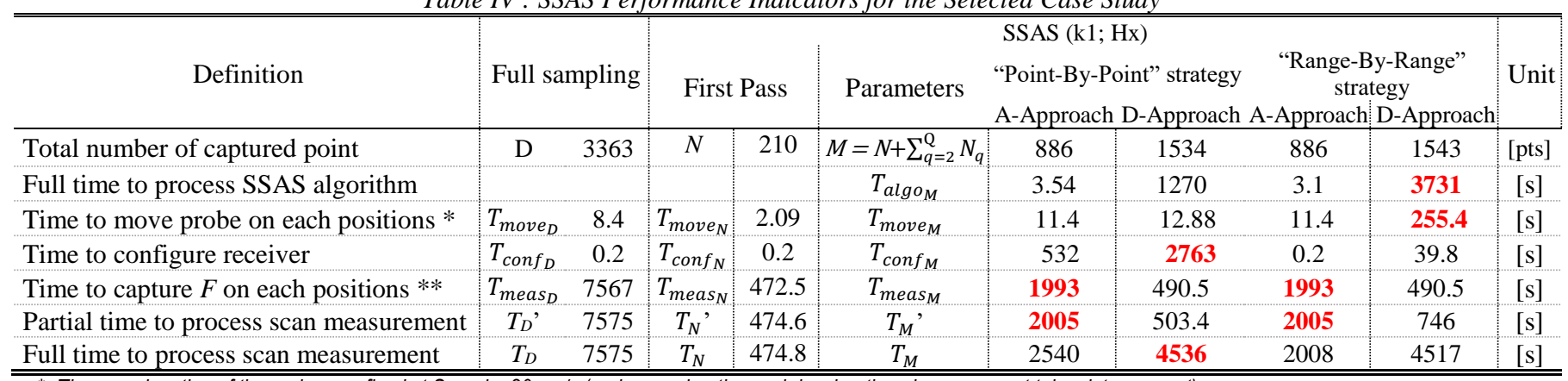

* The speed motion of the probe was fixed at Speed $=20 \mathrm{~cm} / \mathrm{s}$ (probe acceleration and deceleration phases are not taken into account).

** The time to capture all frequency range for one point is fixed at $2.25 \mathrm{~s}$.

proposed approach. The number of points $M$, where at least one frequency value is captured, increases with the segmentation of the frequency range. It must be pointed out however that this result is misleading. In fact a more detailed analysis based on Fig. 6 and the $N_{\text {Freq }}$ Captured $_{M}$ histograms shown in Fig. 7 can be carried out.

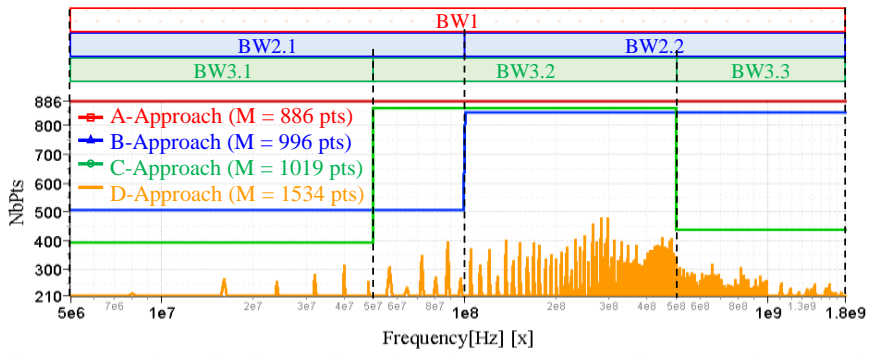

Fig. 6. Number of points collected vs. frequency according to each approach (k1 criterion, Hx component, "Point-By-Point" strategy)

Fig. 7 shows the measurement time evaluation for the "PointBy-Point" strategy according to the four proposed approaches. The result for each field component $(\mathrm{Hx}, \mathrm{Hy}$ and $\mathrm{Hz})$ is displayed respectively in the blue, green and red histogram plots. The part representing the first step, common to each field component, is depicted in the orange box. The results for each $k$ selection criterion are grouped together. Some figures have a right axis to show the relative value of the performance factor according to the full sampling. (e.g. in Fig7, $M$ figure presents on right axis the $M / D$ ratio [\%] where $D$ is the total number of captured point in full sampling measurement).

In Fig. 7, the largest reduction of the $T_{\text {meas }}$ is provided by the D-Approach. It confirms that the frequency segmentation could highly reduce the measurement time with the lowest $T_{M}{ }^{\prime}$ value, where $T_{M}{ }^{\prime}=T_{\text {move }_{M}}+T_{\text {meas }_{M}}$. This value, for $\mathrm{D}$ Approach, represents the optimal time (with optimal $T_{\text {move }}$ and $T_{\text {meas }}$ values) but in reality it cannot be reached. $T_{M}{ }^{\prime}$ considers that the measurement is done without accounting for the equipment configuration time: $T_{\text {conf }}$ (and the time to process the SSAS algorithm $T_{\text {algo }_{M}}$ ).

The sweep time to capture all frequency ranges $(5 \mathrm{MHz}$ to $1.8 \mathrm{GHz}$ ) is just the sum of the time required to capture each frequency sub-range. In real condition, it is necessary to include the time to configure the receiver between each capture of frequency sub-range. In the D-Approach, the impact could be significant. In the following paragraphs, $T_{\text {conf }_{M}}$ and $T_{\text {algo }}$ will be taken into account in the measurement time evaluation.

\section{B. Evaluation of the different measurement strategy}

As presented in the beginning of this chapter, two strategies can be implemented to process the SSAS algorithm. The choice of strategy does not seriously affect the final number of point $M$. Consequently $T_{\text {meas }}$ does not change significantly (this is why Fig. 8 does not display $T_{\text {meas }_{M}}$ values for the « Range-byRange » strategy).

In « Point-by-Point » strategy, each point is processed only one time for all frequency ranges. The main advantage of this strategy is that the displacement between each point is done only once. This strategy yields the optimal $T_{\text {move }_{M}}$ independently from the chosen approach $\left(11 \mathrm{~s}<T_{\text {move }_{M}}<18 \mathrm{~s}\right.$ as shown in Fig. 7). However, $T_{\text {conf }}$ can be seriously increased according to the number of sub-ranges $N_{B W}$. The following equations propose an estimation of $T_{\text {move }_{N Q}}$ and $T_{\text {conf }_{N Q}}$ for the «Point-by-Point » strategy:

$$
\begin{aligned}
\min \left(T_{\text {move }_{N Q}}\right) & =\frac{\sum_{q=2}^{Q}\left[(N q-1) \cdot\left(\frac{W}{\left.{ }_{2}^{q} /{ }^{2}+1\right]}\right)\right]}{\text { Speed }} \\
\max \left(T_{\text {conf }_{N Q}}\right) & =\sum_{q=2}^{Q}(N q) \cdot N_{B W} \cdot T_{c} .
\end{aligned}
$$

In « Range-by-Range » strategy, each frequency sub-range is processed only once. The main advantage of this strategy is that the reconfiguration of equipment is done only once for each frequency range. Although this strategy provides the optimal $T_{\text {conf }}$ value. Conversely, $T_{\text {move }}$ can be seriously increased according to $N_{B W}$. The following equations propose an estimation of $T_{\text {move }_{N Q}}$ and $T_{\text {conf }_{N Q}}$ for the «Range-by-Range » strategy:

$$
\begin{gathered}
\min \left(T_{\text {move }_{N Q}}\right)=N_{B W} \cdot \frac{\sum_{q=2}^{Q}\left[\left(N_{q}-1\right) \cdot\left(\frac{W}{2}\left[\frac{w}{2+1}\right)\right]\right.}{\text { Speed }} \\
\max \left(T_{\text {conf }_{N Q}}\right)=N_{B W} \cdot T_{C} .
\end{gathered}
$$

It should be underlined that the extraction of simple analytical equations, for a precise evaluation of the optimized receiver configuration time and the displacement time, is not straightforward in an optimization process like the SSAS 


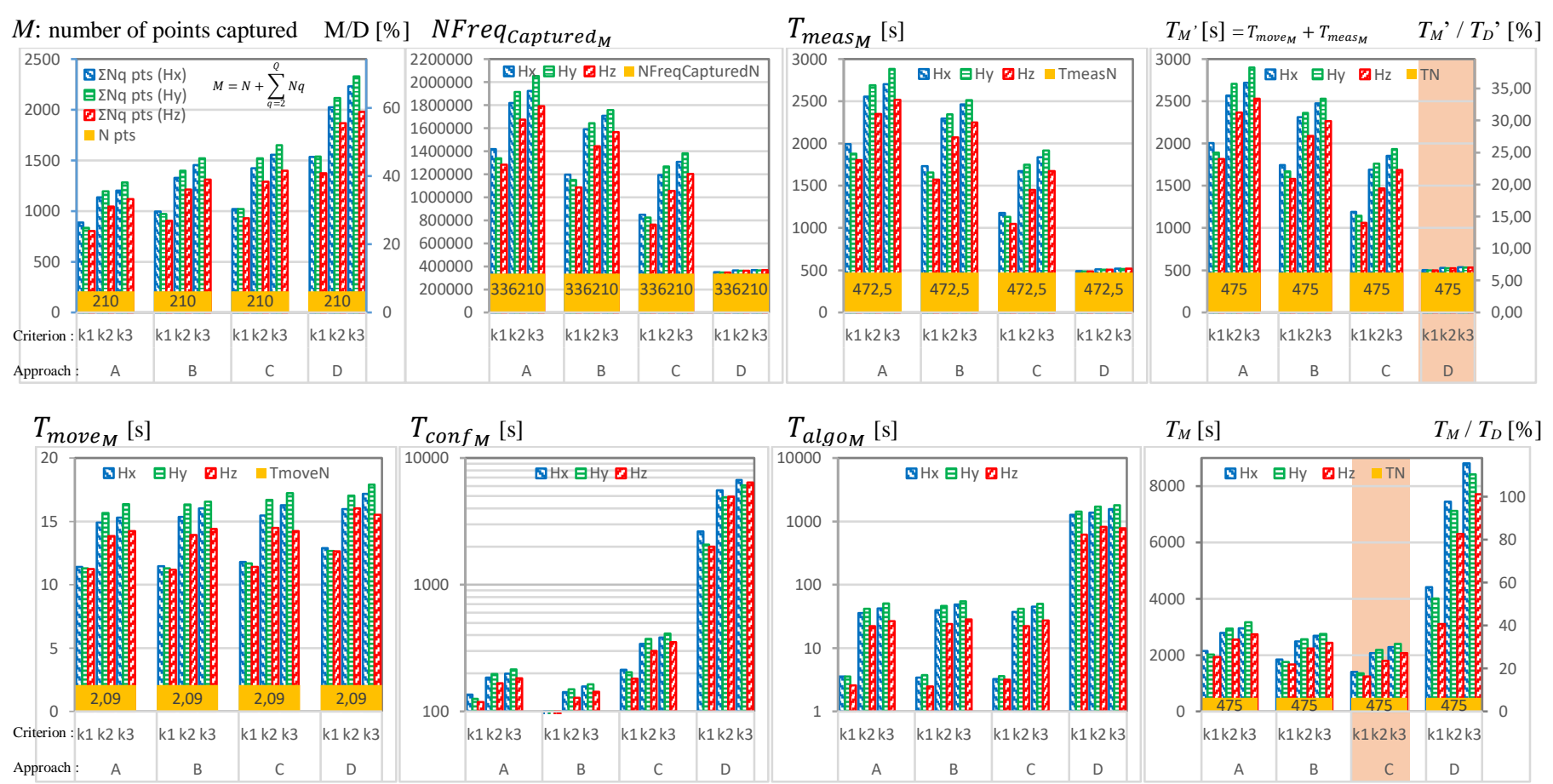

Fig. 7. Performance factor and measurement time evaluation depending on the criteterion and approach using « Point-by-Point » strategy
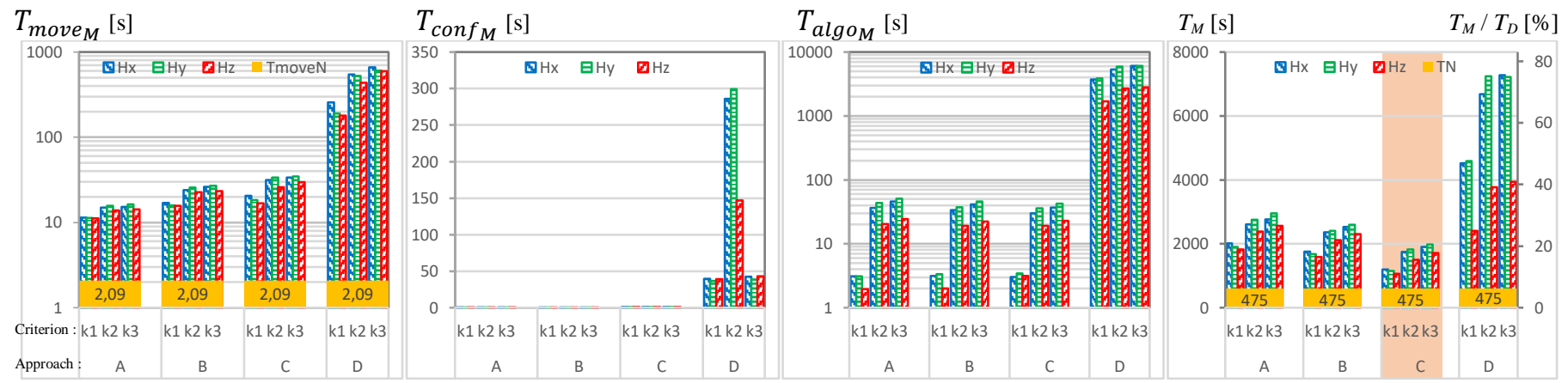

Fig. 8. Measurement time evaluation depending on the criteterion and approach using « Range-by-Range » strategy

algorithm. Furthermore, some optimizations are added to minimize each parameter. For example, about the evaluation of $T_{\text {conf }_{N Q}}$, if the same configuration is sent sequentially to the receiver, the second configuration time is not taken into account. The aim of (24)-(27) equations is to highlight the dependency on the both parameters $N_{B W}$ and $N q$.

Talgo : the time to process the SSAS algorithm can be estimated smaller than $1 \mathrm{~ms}$ per point and per subrange (Talgo is not significantly impacted by $N_{F r e q}$ ). This value depends of the computer configuration (here: 64 Bit Intel@ core i58265U @ $1.6 \mathrm{GHz}$ ). Furthermore, for this study, some computation and data saving are added to the SSAS algorithm in order to collect all performance indicators according to the algorithm configuration. $T_{\text {algo }}$ is given here only for information and to draw the trend.

Noteworthy is that $T_{\text {algo }}$ is proportional to the factor: $M$. $N_{B W}$. Consequently, the D-Approach will yield the worst case regarding the $T_{\text {algo }}$ value.

Fig. 7 shows the results for the « Point-by-Point» strategy. The optimal value of $T_{\text {move }}$ is found independently from the chosen approach. The D-Approach is significantly affected by the $T_{\text {conf }_{M}}$ and the $T_{\text {algo }_{M}} . T_{M}$ value which confirms the bad performance of this approach. The C-Approach yields the best solution. In fact, the measurement time is reduced to $22 \mathrm{~min}$ with the SSAS algorithm (using kl) instead of $2 \mathrm{~h} 06$ min for the full sampling measurement, i.e. a reduction by a factor 6 .

Fig. 8 shows the results for the « Range-by-Range » strategy, which provides the optimal value of $T_{\text {conf }}$. In this case, the CApproach provides again the best results. The full measurement time $T_{M}$ is reduced to $18 \mathrm{~min}$, i.e. a reduction by a factor 7 compared to a full sampling measurement.

Fig. 9 corroborates the choice to limit the number of captured points in order to reduce the full measurement duration.

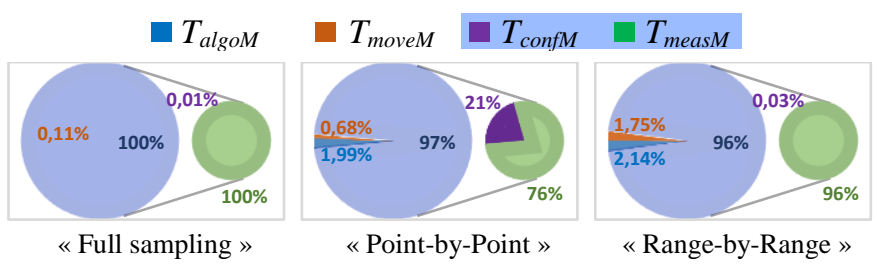

Fig. 9. Relative measurement time evaluation according to the strategy used (C-Approach - Hy - k3)

Independently of the used strategy, $T_{\text {meas }_{M}}$, which is directly 
linked to the number of captured points $M$, accounts for more than $75 \%$ of the full time of measurement (including receiver configuration time). $T_{\text {algo }_{M}}$ and $T_{\text {move }_{M}}$ only represent $4 \%$ of the measurement duration.

\section{Evaluation of the measurement error added by the SSAS algorithm}

The measurement error added by the SSAS algorithm has been discussed in the previous paper [1] for mono-frequency measurement. Excepted for the D-Approach, the frequency range segmentation, introduces additional frequency field captured of $F$ that the selection criterion of the SSAS algorithm would not have selected in its mono-frequency version (DApproach). This added information improves the knowledge about $F$ at the cost of a very slight increase in $T_{\text {meas }_{M}}$. This drawback is largely compensated by the reduction of $T_{\text {conf }}$.

Consequently, the measurement error added by the SSAS algorithm, in multi-frequency context (B-Approach or CApproach) would be at minima equal or lower than that given in our previous paper [1] (D-Approach). Fig. 10 confirms this point. Independently from the used strategy, the C-Approach with $k 3$ criterion yielded a MeanError value closed to $0.5 \mathrm{~dB}$ and a MaxErrorOnLocalMax value closed to $1 \mathrm{~dB}$ (as reminder in mono-frequency version [1], the average estimation error MeanError was less than $1 \mathrm{~dB}$ and the maximum error on the local maxima MaxErrorOnLocalMax was less than $2 \mathrm{~dB}$ ).

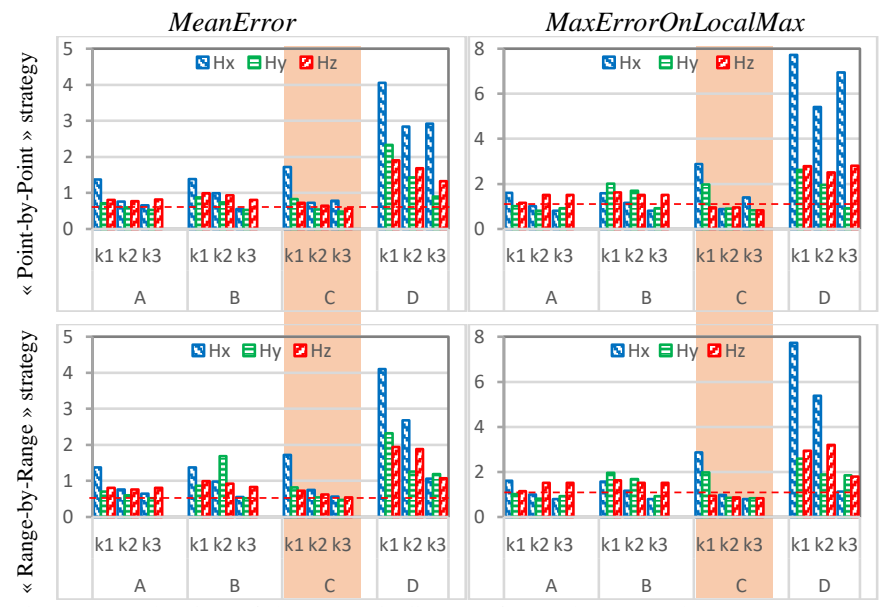

Fig. 10. Evaluation of the errors indicators for each approach

\section{DISCUSSION - CONCLUSION}

Without any acceleration method, excessive measurement duration may limit the practical use of near-field scanning for full analysis of complex electronic devices. This paper proposes a fast near-field measurement method based on the SSAS algorithm. Several strategies and approaches to segment the frequency range of interest have been presented. It demonstrates that a simple analysis could be misleading about the best way to minimize near-field measurement time. A rigorous analysis can be complex since a large number of measurement parameters can influence the performance of the SSAS algorithm. The choice of the best strategy and approach depends on the equipment used for the near-field measurement. The main parameters are the speed of the robot used to move the probe and the speed of the receiver to capture and transfer the data. With a very fast robot and an old receiver (slow moving and data transfer), the best method is to use the « Range-by-Range » strategy along with the A-Approach. This configuration limits the wasted time used to configure the receiver in order to minimize the impact of the slower of receiver. Otherwise, with a slow robot and a powerful receiver, the best method is to use the «Point-by-Point » strategy along with the $\mathrm{C}$-approach. In this configuration, the number of probe displacements is minimized. In other cases, a tradeoff must be found.

Today, robots are quite fast and all receivers implement highspeed communication interface. Thus, the recommended configuration is to use the «Range-by-Range » strategy. The choice of approach or more exactly the choice of the best segmentation of the frequency range is not straightforward. It depends on the DUT characteristics. If the DUT's activity is centered on one spectral source, most likely it is not necessary to segment the frequency range. Otherwise, if the activity of the DUT includes several sources, with spectra scattered on different frequency ranges, it could be more efficient to segment the frequency range. A two harmonic sources case study is used for this work. In this configuration, the greatest time reduction is given by the "Range-by-Range » strategy using the $\mathrm{C}$-approach. For the selected case study, the CApproach proposes to segment the frequency sub-range in three parts. Based on this study, the best segmentation must be fixed to $(X+1)$ frequency range where $X$ is the number of different harmonic sources present on the DUT. This segmentation of the frequency range focuses the measurement only on each specific activity in order to minimize the wasted time to capture the noisy points.

As presented in Fig. 11 (resuming data of the Fig. 7 and Fig. 8), the SSAS algorithm allows a time reduction factor ranging from four up to seven compared to the full sampling measurement (e.g. 18 minutes with the SSAS algorithm instead of 2 hours using the full sampling measurement).

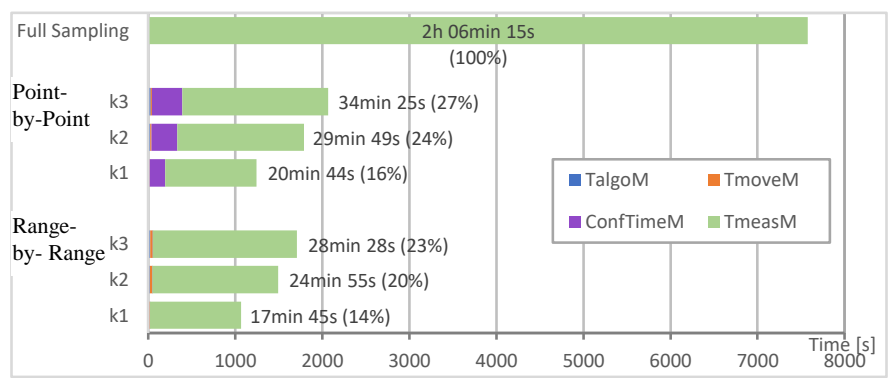

Fig. 11. Time comparaison for each strategy (C-Approach; Hz component)

The working hypothesis retained for this study is to consider only the field magnitude. The $k$ criterion (5) is built on this assumption. The knowledge of the field magnitude is enough during the investigation. However, if near-field measurement is used for numerical application (e.g. modelling of DUT, nearfield to far-field transform), the phase information is mandatory. In this context and following [14], an additional task of this work should be carried out to update the $k$ criterion in order to manage both real and imaginary parts of the field. 


\section{REFERENCES}

[1] S. Serpaud, A. Boyer, S. Ben Dhia, F. Coccetti, "Fast and Accurate NearField Measurement Method Using Sequential Spatial Adaptive Sampling (SSAS) Algorithm", IEEE Trans. On EMC, Early Access, October 2020, doi 10.1109/TEMC.2020.3025547.

[2] M. Sørensen, H. Kajbaf, V. V. Khilkevich, L. Zhang and D. Pommerenke, "Analysis of the Effect on Image Quality of Different Scanning Point Selection Methods in Sparse ESM," in IEEE Transactions on Electromagnetic Compatibility, vol. 61, no. 6, pp. 1823-1831, Dec. 2019, doi: 10.1109/TEMC.2018.2866361.

[3] M. A. Qureshi, C. H. Schmidt, T. F. Eibert, "Adaptive Sampling in Spherical and Cylindrical Near-Field Antenna Measurements," IEEE Antennas and Propagation Magazine 55(1): pp. 243-249, February 2013, DOI. 10.1109/MAP.2013.6474537.

[4] R. Xu,1 X. Zhou, Y. Hirano, R. Tachibana, T. Hara, S. Kido, H. Fujita, "Particle System Based Adaptive Sampling on Spherical Parameter Space to Improve the MDL Method for Construction of Statistical Shape Models," Computational and Mathematical Methods in Medicine, Vol. 2013, pp 9, http://dx.doi.org/10.1155/2013/196259

[5] R. Theunissen1 and P. Gjelstrup, "Adaptive sampling in two dimensions for point-wise experimental measurement techniques," in Proc. 19th Int. Symp. Appl. Laser Imag. Techn. Fluid Mech., Jul. 16-19, 2018, pp. 10351048 .

[6] D. Deschrijver, D. Pissoort, T. Dhaene, "Automated near-field scanning algorithm for the EMC analysis of electronic devices," IEEE Transactions on EMC, vol. 54, no. 3, pp. 502-510, June 2012

[7] T. Dorné et al., "Optimized sequential sampling algorithm for EMI nearfield scanning," in Proc. EMC Eur., Brugge, Sep. 2-6, 2013, pp. 385-388.

[8] J. Urbonas, H. Votsi, A. Shakouri and P. H. Aaen, "Surrogate ModelingBased Acceleration of Multi-Harmonic Near-Field Measurements," 2021 96th ARFTG Microwave Measurement Conference (ARFTG), 2021, pp. 1-5, doi: 10.1109/ARFTG49670.2021.9425147.

[9] S. Serpaud, A. Boyer, and S. Ben Dhia, "Sequential adaptive sampling algorithm to reduce the near-field measurement time," in Proc. EMC-Eur., Sep. 2-6, 2019, pp. 680-685, doi: 10.1109/EMCEurope.2019.8872034.

[10] TS, Ed.1: Integrated Circuits- Measurement of Electromagnetic Emissions, $150 \mathrm{kHz}$ to $1 \mathrm{GHz}$ - Part 3: Measurement of Radiated Emissions-Surface Scan Method, IEC 61967-3.

[11] A. Brandt. "Multi-level adaptive solutions to boundary-value problems," Mathematics of Computation, vol. 31, pp 333-390, 1977.

[12] Z. Hong et al., "Electromagnetic Pattern Extraction and Grouping for Near-Field Scanning of Integrated Circuits by PCA and K-Means Approaches," in IEEE Transactions on Electromagnetic Compatibility, vol. 61, no. 6, pp. 1811-1822, Dec. 2019, doi: 10.1109/TEMC.2018.2890026.

[13] S. Serpaud, C. Ghfiri, A. Boyer and A. Durier, "Proposal for combined conducted and radiated emission modelling for Integrated Circuit," 2017 11th International Workshop on the Electromagnetic Compatibility of Integrated Circuits (EMCCompo), St. Petersburg, 2017, pp. 172-177, doi: 10.1109/EMCCompo.2017.7998105.

[14] T. Claeys, D. Pissoort, D. Deschrijver, I. Couckuyt and T. Dhaene, "Sequential sampling algorithm for simultaneous near-field scanning of amplitude and phase," 2014 International Symposium on Electromagnetic Compatibility, 2014, pp. 79-84, doi: 10.1109/EMCEurope.2014.6930881.

[15] The Python Scikit library v0.21.3-2019.[Online] - https://scikit-learn.org

[16] IC-EMC 2v9 freeware - 2020.[Online] - https://www.ic-emc.org

[17] Case studies data - 2020.[Online] - http://ic-emc.org/nefisc-editor.html

[18] RTCA DO-160 - Section 21.0 - Emission of Radio Frequency Energy

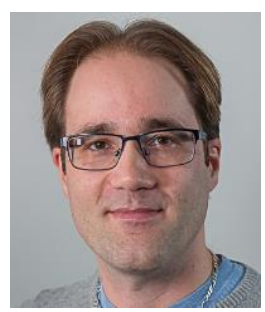

Sébastien Serpaud (Student Member, IEEE) received the engineering professional qualification in Electronic and Computer Science and the Master's degree in Microelectronic and Microsystems circuit design in 2006 from the National Institute of Applied Sciences (INSA) of Toulouse, France.

In 2006, he joined Freescale Semiconductor in research activity about EMC for semiconductors manufacturing. From 2007 to 2017, he was an
EMC research engineer with Nexio. In 2015, he joined IRT SaintExupéry institute, Toulouse, France, where he is currently a team leader of the research group on EMC field. Since 2018, he has been working toward the Ph.D. degree at INSA, Toulouse, France, in application of the near-field emission measurement method to improve the design and the investigation of EMC. His research interests include development of investigation tools around the near-field measurement, the EMC modeling at IC and electronic board level, and freeware tools development for EMC (NeFiSc Editor and Touchstone Editor) http://emctools.free.fr.

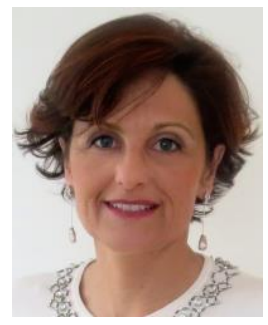

Sonia Ben Dhia obtained her Masters degree in electrical engineering in 1995, and a Ph.D. in Electronic Design from the Institut National des Sciences Appliquées (INSA), Toulouse, France, in 1998. Full professor at INSA-Toulouse (French engineering institute), Department of Electrical and Computer Engineering, she teaches digital electronics, IC testability and reliability, and analog and RF CMOS design. CEO of INSA Euro-Méditerranée, Fès, Morocco (2014-2017), she was responsible for the overall leadership and management of this new engineering institute. Her research interests at LAAS - CNRS Toulouse include signal integrity in nano-scale CMOS ICs, electromagnetic compatibility and reliability of ICs. She has authored and co-authored 3 books, more than 120 publications in peer-reviewed journals \& conference proceedings and supervised $14 \mathrm{PhD}$ theses and 9 M.Sc. theses.

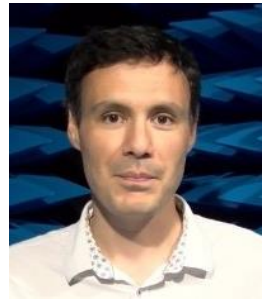

Alexandre Boyer obtained a Masters degree in electrical engineering in 2004 and a PhD in Electronics from the Institut Nationale des Sciences Appliquées (INSA) in Toulouse, France, in 2007. He is currently an Associate Professor in the Department of Electrical and Computer Engineering at INSA, Toulouse. He is leading his research at the Laboratoire d'Analyse et d'Architecture des Systèmes (LAAS-CNRS), as part of the 'Energy and Embedded Systems' research group. His current research interests include EMC measurements, IC EMC and reliability modeling, and computer aided design (CAD) tool development for EMC (IC-EMC freeware).

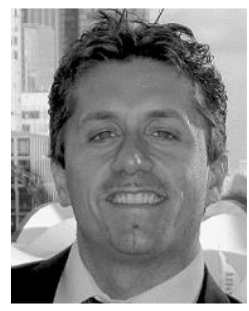

Fabio Coccetti received the Laurea M.S. degree in electrical engineering from the University of Perugia, Perugia, Italy, the Ph.D. degree in high frequency engineering at the Technische Universitt Mnchen (TUM) in Munich Germany, and the Habilitation direction de recherche (HDR) from INP-Toulouse, in 1999, 2004, and 2013, respectively.

He has been visiting scientist at University of Michigan USA in 2000 and from 2004 to 2008, he has been working as research scientist at the Laboratoire d'Analyse et d'Architectures des Systèmes at the Centre National de la Recherche Scientifique (LAAS-CNRS) in Toulouse, France. From 2008 to 2016 he has been project manager at Elemca (Toulouse) and from 2016 to 2018 general manager of RF Microtech France. Since March, he joined the IRT Saint Exupery as head of the Component Modeling and Reliabilty competence center within the More Electrical Aircraft Department. Dr. Coccetti has been involved in numerous research projects going from the theoretical and experimental investigation of power handling in RFMEMS to design modelling and experimental validation of reliability for Microelectronics, nanosystems and more recently power electronics.

Dr. Coccetti has more than 150 publications in peer reviewed conference and journal papers, and he is co-author $s$ of two book chapters. He has been European Microwave Lecturer (2013-2016) and member of the European Microwave Association (EUMA). He is member of the IEEE society, cofounder and chair of the RF Nanotechnology Committee within the IEEE-MTT society. He was the recipient of the IHP International Wolfgang Mehr Fellowship Award in 2016. 